\title{
BMJ open Statin use and clinical outcomes in older men: a prospective population-based study
}

\author{
Danijela Gnjidic, ${ }^{1,2,3,4}$ David G Le Couteur, ${ }^{2,4,5}$ Fiona M Blyth, ${ }^{2,4}$ Tom Travison, ${ }^{6}$ \\ Kris Rogers, ${ }^{7}$ Vasi Naganathan, ${ }^{2,4}$ Robert G Cumming, ${ }^{2,4,8}$ Louise Waite, ${ }^{2,4}$ \\ Markus J Seibel, ${ }^{4,5}$ David J Handelsman, ${ }^{4,5}$ Andrew J McLachlan, ${ }^{1,2}$ Sarah N Hilmer ${ }^{3,4}$
}

To cite: Gnjidic D, Le Couteur DG, Blyth FM, et al. Statin use and clinical outcomes in older men: a prospective population-based study. BMJ Open 2013;3 e002333. doi:10.1136/ bmjopen-2012-002333

- Prepublication history for this paper are available online. To view these files please visit the journal online (http://dx.doi.org/10.1136/ bmjopen-2012-002333).

Received 12 November 2012 Revised 29 January 2013 Accepted 31 January 2013

This final article is available for use under the terms of the Creative Commons Attribution Non-Commercial 2.0 Licence; see http://bmjopen.bmj.com

For numbered affiliations see end of article.

\section{Correspondence to} Dr Danijela Gnjidic; danijela.gnjidic@sydney.edu.au

\section{ABSTRACT}

Objective: The aim of this analysis was to investigate the relationship of statins with institutionalisation and death in older men living in the community, accounting for frailty.

Design: Prospective cohort study.

Setting: Community-dwelling men participating in the Concord Health and Ageing in Men Project, Sydney, Australia.

Participants: Men aged $\geq 70$ years ( $n=1665)$.

Measurements: Data collected during baseline assessments and follow-up (maximum of 6.79 years) were obtained. Information regarding statin use was captured at baseline, between 2005 and 2007. Proportional hazards regression analysis was conducted to estimate the risk of institutionalisation and death according to statin use (exposure, duration and dose) and frailty status, with adjustment for sociodemographics, medical diagnosis and other clinically relevant factors. A secondary analysis used propensity score matching to replicate covariate adjustment in regression models.

Results: At baseline, $43 \%$ of participants reported taking statins. Over 6.79 years of follow-up, 132 $(7.9 \%)$ participants were institutionalised and 358 $(21.5 \%)$ participants had died. In the adjusted models, baseline statin use was not statistically associated with increased risk of institutionalisation ( $\mathrm{HR}=1.60 ; 95 \% \mathrm{Cl} 0.98$ to 2.63$)$ or death (HR=0.88; $95 \% \mathrm{Cl} 0.66$ to 1.18 ). There was no significant association between duration and dose of statins used with either outcome. Propensity scoring yielded similar findings. Compared with non-frail participants not prescribed statins, the adjusted HR for institutionalisation for non-frail participants prescribed statins was $1.43(95 \% \mathrm{Cl} 0.81$ to 2.51$)$; for frail participants not prescribed statins, it was $2.07(95 \% \mathrm{Cl}$ 1.11 to 3.86$)$ and for frail participants prescribed statins, it was $4.34(95 \% \mathrm{Cl} 2.02$ to 9.33$)$.

Conclusions: These data suggest a lack of significant association between statin use and institutionalisation or death in older men. These findings call for real-world trials specifically designed for frail older people to examine the impact of statins on clinical outcomes.

\section{ARTICLE SUMMARY}

\section{Article focus}

- Evidence from randomised trials supports the benefits of statins in reducing cardiovascular morbidity and mortality.

- There are limited data in relation to statin use and clinical outcomes in representative populations of community-dwelling older people.

Key messages

- In this prospective cohort study, there was no significant association between statin use and institutionalisation or death in community-dwelling older men.

- Frail men were more likely to be institutionalised and die than non-frail men, independent of their statin exposure.

- Randomised trials utilising operational frailty definitions with clinically relevant endpoints are required to inform therapy in this population.

Strengths and limitations of this study

- This is a prospective cohort study of communitydwelling older men, with rich data sources.

- The study may have been underpowered to demonstrate the statistical significance in relation to statin use and institutionalisation.

- Observational studies of preventative medication users, including statins, are often biased by healthy user and a healthy tolerator bias.

\section{INTRODUCTION}

Statins, 3-hydroxy-3-methylglutaryl coenzyme A reductase inhibitors are commonly used medicines in older people. In a recent Australian study, $43 \%$ of community-dwelling people aged $\geq 75$ years reported using statins. ${ }^{1}$ The benefits of statins in relation to primary and secondary prevention of cardiovascular morbidity and mortality have been demonstrated in a number of randomised clinical trials (RCTs). ${ }^{2}{ }^{3}$ However, it is not clear how the findings of these trials translate to clinically significant outcomes in general 
populations of older people, as the representation and representativeness of older people in published RCTs of statins generally poor. ${ }^{4}$ Therefore, observational studies are often essential to elucidate the intended effects of medicines in this population. ${ }^{5}$ Moreover, the benefit to harm ratio of medicines is altered in older adults due to comorbid conditions, age-related physiological changes, increased risk of adverse drug reactions and multiple medicines. ${ }^{6}$

The pharmacological response to medicines is further altered when older individuals become frail. ${ }^{7}$ Frailty is a geriatric syndrome associated with functional impairment and increased vulnerability to disease, disability and mortality in older people. ${ }^{8}$ Frail individuals are more likely to use more medicines, ${ }^{9}$ and are at increased risk of adverse effects from medicines. ${ }^{7}$ Conversely, frail older people are less likely to be recruited to and participate in RCTs. ${ }^{7}$ Currently, there are limited data to guide prescribing to minimise medication-related harms in older people with geriatric syndromes including frailty. Moreover, evidence on clinically relevant outcomes of Drug-Geriatric Syndrome Interactions (DGSI) in older adults who have already developed a geriatric syndrome is limited. ${ }^{10}$ It is unknown whether medicines do more good than harm in older adults with established geriatric syndromes.

There have been mixed findings across observational studies investigating the associations between statin use and geriatric syndromes and physical performance measures in older people. Statins have been associated with faster walking speed in patients with peripheral arterial or vascular disease. ${ }^{11} 12$ In contrast, a recent study reported no association of statin use with mobility in older community-dwelling people. ${ }^{13}$ In a study of community-dwelling older women, current statin use was not associated with incident frailty over 3 years. ${ }^{14}$ Statins in older people may increase the risk of both institutionalisation and death by causing myopathy or muscle damage. ${ }^{15}$ Recent evidence also suggests that statins have adverse effects of energy and fatigue with exertion. ${ }^{16}$ Statin-related myopathy is likely to have a greater impact on frail older adults with limited musculoskeletal reserve than on younger people who generally have more muscle mass and strength.

While the data from published RCTs and prospective studies indicate that statins reduce the incidence of cardiovascular events, ${ }^{17}$ there are still significant gaps in evidence on the safety of statins in a real-world setting. To our knowledge, no study has examined the association between the use of statins and institutionalisation in a representative population of older people or in frail older people. Moreover, the evidence on the impact of interactions between statins and frailty (DGSI) on clinical outcomes in older people is yet to be established. The objectives of this study were to investigate the relationship between statin use and the interactions between statins and frailty with institutionalisation and death in community-dwelling older men living in Sydney, Australia.

\section{METHODS}

\section{Study population}

Participants were community-dwelling men enrolled in the Concord Health and Ageing in Men Project (CHAMP), Sydney, Australia. ${ }^{18}$ Eligible participants were $\geq 70$ years and living in a specific study area. The only exclusion criterion was living in a residential aged care facility (RACF). Electoral Roll was chosen as the sampling frame for the study. In Australia, registration on the Electoral Roll is compulsory and regularly updated, making it a suitable population-wide sampling frame. Men were recruited during 2005-2007. Of the 2815 eligible men contacted, 1511 (53.7\%) participated in the study. An additional 194 (11.4\%) men living in the study area heard about the study from friends or the local media and were recruited before receiving an invitation letter, giving a final sample of 1705 participants. Participants underwent baseline assessments which comprised self-completed study questionnaires and a clinical assessment that consisted of physical performance measures, neuropsychological testing, biological measures and medication inventory. Participants also agreed to be contacted every 2 years subsequently for follow-up assessment. After exclusion for missing data $(n=40)$, a total of 1665 men were included in the analysis (figure 1).

\section{Medication assessment and classification of statin exposure}

A medication inventory was conducted of each participant by trained personnel during the baseline clinic visit. Participants were instructed to bring all the prescription and over-the-counter medications they were taking to the clinic visit for review. They were also asked

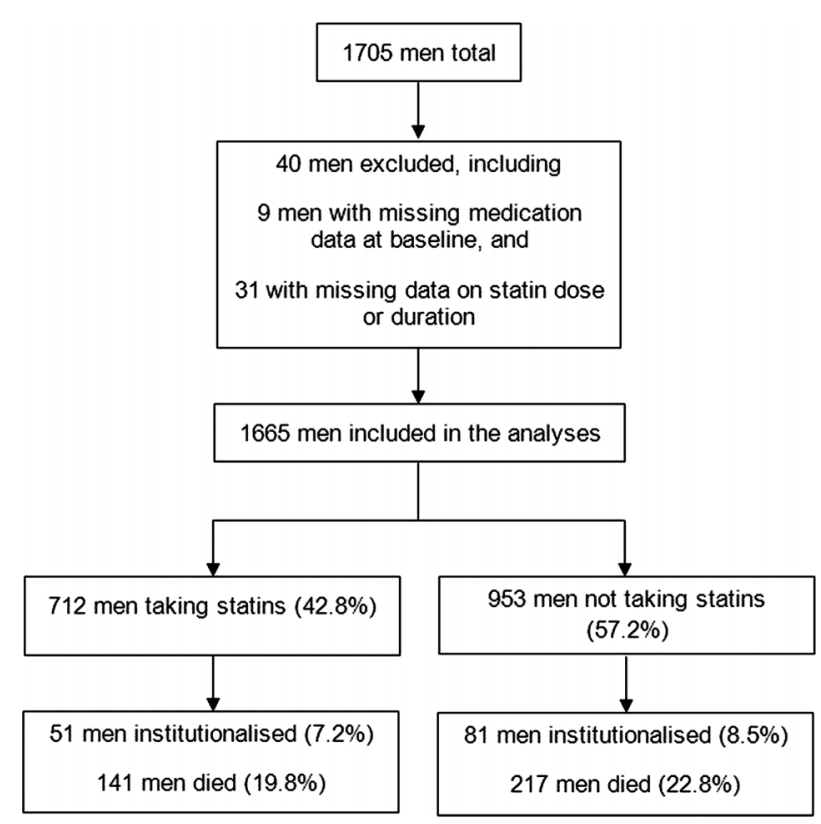

Figure 1 Flowchart for participants taking statins and institutionalisation and death in the Concord Health and Ageing in Men Project. 
whether they had taken any prescription or nonprescription medications during the past month. Details of all medications and prescription patterns were recorded. Reported medicines were coded using the Iowa Drug Information Service code numbers.

Statin baseline exposure was defined using three approaches. We categorised participants as 'statin users' versus 'non-users'. Data on the duration of statin use (years) were obtained and participants were dichotomised at the upper quartile ( $<4$ vs $\geq 4)$. Statin users were characterised using the units of an equivalent dose, indicating the potency of lipid-lowering effects from clinical trials. ${ }^{19} 20$ The daily dose of each statin was converted to an equivalent statin dose based on a lipid-lowering effect of $10 \mathrm{mg}$ of atorvastatin (equivalent to $5 \mathrm{mg}$ of rosuvastatin, $20 \mathrm{mg}$ of simvastatin, $40 \mathrm{mg}$ of lovastatin, $40 \mathrm{mg}$ of pravastatin and $80 \mathrm{mg}$ of fluvastatin). Statin users were grouped into three categories, based on the data distribution, as receiving a low (equivalent dose $<2$ ), medium (equivalent dose 2-4) and high (equivalent dose $\geq 4$ ) statin dose.

\section{Study outcomes}

Data on institutionalisation and death were regularly updated by telephone contact with the participants or their nominated contact person at 4-monthly intervals. Men who were not contactable by telephone were sent letters at 4-monthly intervals. Institutionalisation was defined as entry into a nursing home facility or hostel at any time during the follow-up period of 6.79 years (average 4 years). In Australia, there are two main forms of RACFs: low-level care facilities (hostels) and high-level care facilities (nursing homes). Self-care retirement villages are not considered to be RACFs and residents are not considered 'institutionalised'. Moreover, institutionalisation in Australia is nearly always permanent rather than short-term admission for rehabilitative care after surgery or medical illness. For death outcome, if men withdrew from the study but agreed to passive follow-up, the New South Wales Registry of Births, Deaths and Marriages was contacted to ascertain any deaths. Follow-up began at the baseline assessment and ended on the date of death or the end of the study period. For withdrawals, the end date was the date at which the contact with the death registry was made.

\section{Covariates}

Data on clinically relevant covariates that may influence the association between statin use and outcomes were obtained. ${ }^{13} 14$ Demographic variables included age, education and marital status. Data on the country of birth were obtained and participants were categorised as Australian-born, overseas-born from an English-speaking background (ESB) and overseas-born from a non-ESB. For those who had consumed at least 12 alcoholic drinks in the past year, the frequency and quantity of alcohol consumption was assessed, and men were categorised as safe drinkers (1-21 alcoholic drinks per week) or harmful drinkers ( $>21$ alcoholic drinks per week). Participants who were current non-drinkers were characterised as either 'lifelong abstainers' or 'ex-drinkers'. Tobacco smoking status (allocated as 'never smoker', 'ex-smoker' or 'current smoker') was also assessed.

Data on cardiovascular diseases (CVDs) including hypertension, coronary artery disease or myocardial infarction, angina and congestive heart failure were obtained. The number of CVD diseases was dichotomised at the upper quartile ( $\leq 1$ vs $\geq 2$ ). Other medical conditions included: diabetes, thyroid dysfunction, osteoporosis, Paget's disease, stroke, Parkinson's disease, epilepsy, intermittent claudication, chronic obstructive lung disease, liver disease, chronic kidney disease or renal failure, cancer (excluding non-melanoma skin cancers) or arthritis. The number of reported comorbidities was dichotomised at the upper quartile $(\leq 1$ vs $\geq 2$ ). Data on body mass index (BMI, $\mathrm{kg} / \mathrm{m}^{2}$ ) were obtained. Multiple medication use or polypharmacy was defined as the use of $\geq 5$ regular prescription medicines. ${ }^{21}$ Corrected visual acuity was assessed using a Bailey-Lovie chart $\left(<6 / 19\right.$ indicating poor vision). ${ }^{22}$ Data on selfrated health were obtained and dichotomised into excellent/good versus fair/poor/very poor. Depressive symptoms were assessed with the 15-item Geriatric Depression Scale ( $\geq 5$ indicative of depressive symptoms). ${ }^{23}$ Blood samples were drawn after overnight fasting. Total cholesterol, high-density lipoprotein (HDL) cholesterol and triglyceride concentrations were obtained and analysed as continuous variables.

All participants were screened for cognitive impairment, and those who tested positive underwent full neuropsychological assessment. Participants were classified as cognitively impaired if they were diagnosed with either dementia or mild cognitive impairment. ${ }^{24}$ Functional status was measured with Activities of Daily Living (ADL) and Instrumental Activities of Daily (IADL) scales. Disability in ADL and IADL was defined as needing help with $\geq 1$ activity. ${ }^{25}{ }^{26}$ Frailty in this population, described in detail elsewhere, ${ }^{27} 28$ was defined according to the criteria used in the Cardiovascular Health Study (CHS): weight loss/shrinking, weakness, exhaustion, slowness and low activity. ${ }^{8}$ For the weakness and slowness components, the same criteria as in the CHS were applied. Adapted criteria were used for weight loss, exhaustion and low activity as the exact measurements used in the CHS were not available in this study. ${ }^{27}{ }^{28}$ Participants were considered frail if they had three or more frailty criteria, intermediate (pre-frail) with one or two criteria and robust (not-frail) without any criteria.

\section{Statistical analysis}

Data are summarised as means (SDs) or counts (proportions). Differences in baseline characteristics between statin users and non-users were compared using the nonparametric or $\chi^{2}$ tests as appropriate. Univariate analyses of the association between the various study measures and outcomes were conducted using Log-rank tests and 
examination of survival curves. Tests for linear trends were performed for continuous variables to determine the linearity of their relationship with institutionalisation and death, and to determine whether to enter these variables into models as continuous or categorical variables. The appropriate parameterisation of continuous variables as either categorical or continuous was also confirmed in the final model by using Akaike's Information Criterion. Univariate Cox regressions were conducted to determine the unadjusted HR with $95 \%$ CI for the effects of statins on institutionalisation and death. We then conducted the Cox proportional hazards regression models for the effects of statins on institutionalisation and death, and adjusted for all potential confounding factors at baseline including age, education, marital status, alcohol use, smoking, BMI, self-reported comorbidities, self-reported CVDs, impaired vision, depression, cognitive impairment, functional status, self-rated health, polypharmacy, total cholesterol, HDL cholesterol and triglyceride concentrations. These analyses were performed for all different categories of statin exposure.

The propensity score analysis was performed to minimise the effects of covariates in the evaluation of the association between statin use and institutionalisation and death. ${ }^{29}$ Participant-specific propensity scores were estimated from a logistic regression model to predict the probability of statin prescription. All covariates were considered in the logistic regression model. The association between statins and institutionalisation and death was evaluated in Cox regressions models after adjusting for the estimated propensity score as a continuous and stratified (grouped into quintile) variable. Moreover, as older individuals with geriatric syndromes may have a higher risk for either institutionalisation or death, we conducted subgroup analysis. We stratified participants based on frailty status and statin use as robust or pre-frail not on statins; robust or pre-frail on statins; frail not on statins and frail on statins. Robust or prefrail participants are referred to as "non-frail" in the analysis. We also tested for interaction to assess whether statin effects differed in frail and non-frail men. Data were analysed using SAS V.9.3 (SAS Institute Inc, Cary, North Carolina, USA). The Kaplan-Meier survival curves were generated using SPSS software V.19.0 (SPPS Inc, Chicago, Illinois, USA).

\section{RESULTS}

The baseline characteristics according to statin use are presented in table 1 . The mean (SD) age of participants was 79.6 (5.5) years. At baseline, 743 (42.9\%) participants were identified as taking a statin. Statin users were younger $(p=0.04)$, had more CVD comorbidities $(p<0.0001)$, used more medications $(p<0.0001)$, had higher BMI $(\mathrm{p}<0.0001)$, and were less likely to report good or excellent health $(\mathrm{p}=0.003)$.In this population, $17 \%$ of participants reported taking statins for $<4$ years, and $26 \%$ for $\geq 4$ years. In relation to the statin dose, $17 \%$ were taking low statin doses, $15 \%$ medium doses and
$10 \%$ high statin doses. Over 6.79 years of follow-up, 132 $(7.9 \%)$ participants were institutionalised and 358 (21.5\%) participants had died. Figure 2 shows the Kaplan-Meier survival curves for institutionalisation and death according to the reported statin exposure and frailty status at baseline. There was a significant difference between the groups in time to institutionalisation or death.

Table 2 summarises the results of the Cox regression models. In the adjusted models, baseline use of statins was not significantly associated with increased risks of institutionalisation ( $\mathrm{HR}=1.60 ; 95 \%$ CI 0.98 to 2.63$)$ or death $(\mathrm{HR}=0.88 ; 95 \%$ CI 0.66 to 1.18$)$. Medium $(\mathrm{HR}=2.00 ; 95 \%$ CI 1.02 to 3.93$)$ and high $(\mathrm{HR}=2.45$; 95\% CI 1.12 to 5.33) dose statin users were significantly more likely to be institutionalised when compared with those not taking statins. There was no association between the duration or dose of statins and death. The propensity score-adjusted HR were not significantly altered apart from the association of statin doses with institutionalisation (table 3). In the propensity score-adjusted models, the current use of statins was not significantly associated with institutionalisation ( $\mathrm{HR}=1.43 ; 95 \%$ CI 0.87 to 2.34$)$ or death $(\mathrm{HR}=0.82$; 95\% CI 0.61to 1.10). Medium or high dose statin use was not significantly associated with a higher risk of institutionalisation compared with non-users.

The HRs for institutionalisation and death in frail versus non-frail men, according to statin use, are presented in table 4. Using non-frail men who were not taking statins as the reference group at baseline, non-frail men prescribed statins had an adjusted HR of 1.43 (95\% CI 0.81 to 2.51) for institutionalisation, frail participants not taking statins had an adjusted HR of 2.07 (95\% CI 1.11to 3.86) and frail participants prescribed statins had an HR of 4.34 (95\% CI 2.02 to 9.33) for institutionalisation. Frail participants prescribed statins had an HR of 1.24 (95\% CI 0.71 to 2.17) for death compared with non-frail participants not prescribed statins. However, among men not using statins, frail participants had an HR of 1.53 (95\% CI 1.03 to 2.28) for mortality compared with non-frail participants. In the adjusted models, there was no significant interaction between frailty and statin use with respect to institutionalisation $(\mathrm{p}=0.40)$ or mortality $(\mathrm{p}=0.73)$.

\section{DISCUSSION}

The objective of this cohort analysis was to evaluate the relationship between statins and two clinically important outcomes, institutionalisation and death in older men, accounting for frailty. The main finding of this prospective observational study of community-dwelling older men is the lack of independent association between the use of statins and institutionalisation or death. However, in this population, frailty was associated with higher risks of institutionalisation and death. Frail men were approximately two times more likely to be institutionalised and 
Table 1 Characteristics of 1665 study participants according to baseline reported use of statins

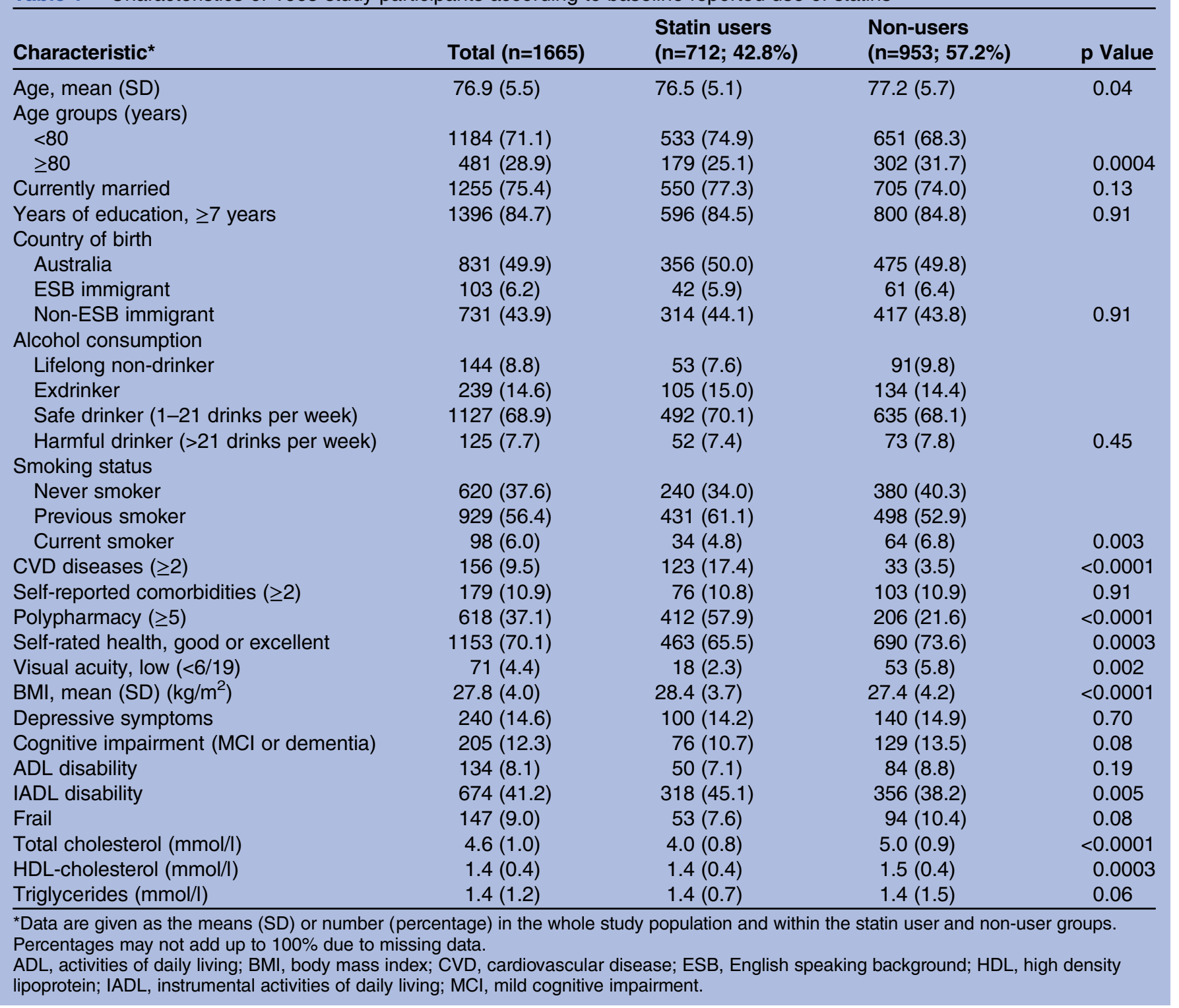

die over 6.8 years of follow-up compared with non-frail men, regardless of their medication exposure.

The prevalence of statin use in this population is comparable to recent studies ${ }^{130}$ but much higher than that reported in studies of older people recruited in the 1990s. In a study of older disabled women in the USA recruited during 1993-1998, ${ }^{14}$ the prevalence of statin use was $8.4 \%$ compared with $12.9 \%$ in a community-dwelling sample of older people enrolled in the Health Ageing and Body Composition study during 1997-1998. ${ }^{13}$ There are no studies conducted in older people that have investigated the association of statin use with institutionalisation. Some studies have showed that statins improve physical function and walking speed ${ }^{11}$ but do not lower the risk of incident frailty over 3 years. ${ }^{14}$ Better performance on functional measures is protective against institutionalisation and death. ${ }^{31}{ }^{32}$ In our study, statin users had an HR of 1.60 (95\% CI 0.98 to 2.63) for increased risk of institutionalisation. Interestingly, high dose statin users had an HR of 2.45 (95\% CI 1.12 to 5.33) for increased risk of institutionalisation. However, this association was not significant in the propensity score adjusted model. Future studies conducted in larger populations are needed to investigate associations between statins and institutionalisation in older people. In relation to statins and mortality, among older people with diabetes living in the community, statin use has been associated with a reduced risk of cardiovascular and all-cause mortality. $^{33}$ In contrast, the Prospective Study of Pravastatin in the Elderly at Risk trial data demonstrates benefits in reducing the risks of coronary diseases; however, there are no benefits in overall mortality. ${ }^{34}$

Moreover, frailty has been associated with an increased risk of institutionalisation ${ }^{35}$ and death. ${ }^{36}$ In this sample, frail men were more likely to be institutionalised and die than non-frail men, irrespective of their statin exposure. 

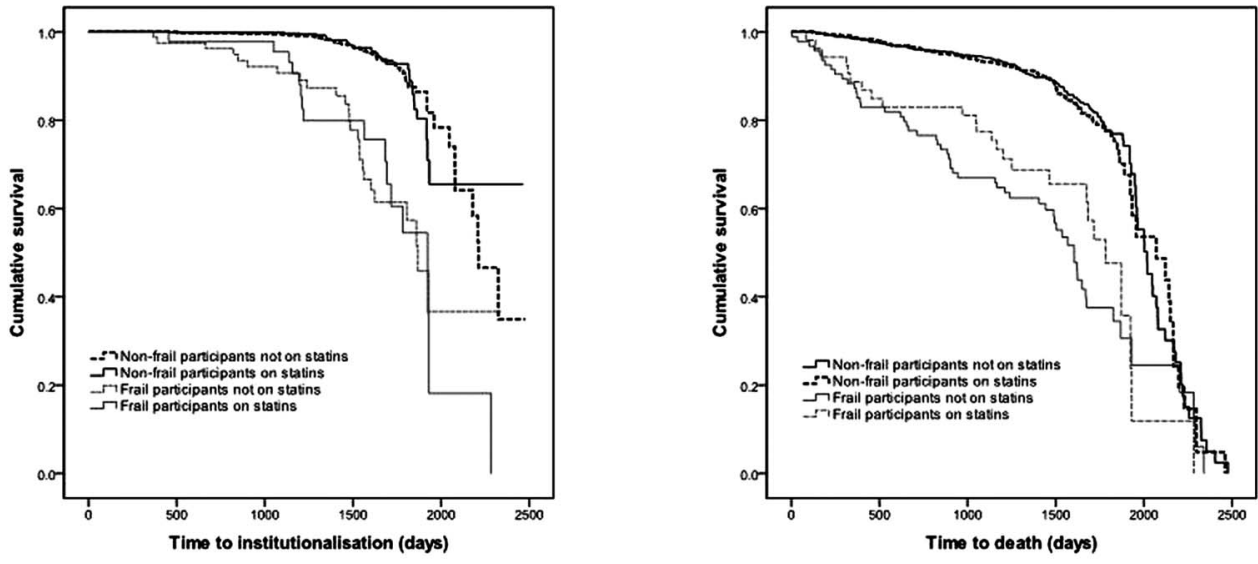

\begin{tabular}{|lcccc|}
\hline & $\begin{array}{c}\text { Non-frail participants not on } \\
\text { statins }\end{array}$ & $\begin{array}{c}\text { Non-frail participants on } \\
\text { statins }\end{array}$ & $\begin{array}{c}\text { Frail participants not on } \\
\text { statins }\end{array}$ & Frail participants on statins \\
$\mathrm{N}=1631$ & 836 & 648 & 94 & 53 \\
Institutionalised & $50(6.0 \%)$ & $34(5.3 \%)$ & $25(26.6 \%)$ & $16(30.2 \%)$ \\
Dead & $149(17.8)$ & $115(17.8 \%)$ & $56(59.6 \%)$ & $25(47.2 \%)$ \\
\hline
\end{tabular}

Figure 2 Kaplan-Meier survival curves for the time until institutionalisation (log-rank test, $p<0.0001)$ and death (log-rank test, $p<0.0001$ ) by reported statin exposure and frailty.

Even though there was no significant interaction between statin use and frailty on institutionalisation rates, frail men using statins had twice the risk of institutionalisation as frail men not using statins. These findings suggest that statins in frail older men may not reduce the risk of institutionalisation or death. Studies with larger numbers of frail participants are needed to estimate the risks of statins in frail older people.

This study has several strengths including the prospective design, good quality medication and outcome data, and adjustment for a number of covariates related to the risk of institutionalisation and death. A careful and systematic medication inventory was performed by checking all medications brought in by the men during a clinic visit. Frailty was ascertained using the validated scale. ${ }^{37}$ We also performed sensitivity analysis including propensity score analysis and stratification of statin users according to their frailty status. While there are different propensity score models that can be used to balance measured covariates, the covariate propensity score adjustment has the best performance for estimating relative risks. ${ }^{38}$

However, there are important limitations to this study. The possibility of confounding by indication and unmeasured confounders needs to be acknowledged, as with any other observational study. Participants with CVDs would be more likely to be prescribed statins and among those with CVDs, those with more CVDs and

Table 2 Association between reported statin use at baseline and institutionalisation and death

\begin{tabular}{|c|c|c|c|c|}
\hline \multirow[b]{2}{*}{ Categorisation of statin use } & \multicolumn{2}{|c|}{ Unadjusted HR (95\% Cl) (n=1665) } & \multicolumn{2}{|c|}{ Adjusted HR $(95 \% \mathrm{Cl})^{\star}(\mathrm{n}=1497)$} \\
\hline & Institutionalisation & Death & Institutionalisation & Death \\
\hline \multicolumn{5}{|l|}{ Statin exposure } \\
\hline Non-users $†$ & 1.00 & 1.00 & 1.00 & 1.00 \\
\hline Users & 0.90 (0.63 to 1.27$)$ & $0.93(0.75$ to 1.15$)$ & 1.60 (0.98 to 2.63$)$ & $0.88(0.66$ to 1.18$)$ \\
\hline \multicolumn{5}{|l|}{ Duration of statin use } \\
\hline Non-users & 1.00 & 1.00 & 1.00 & 1.00 \\
\hline $0-<4$ years & $1.10(0.71$ to 1.68$)$ & $0.87(0.65$ to 1.16$)$ & $1.73(0.97$ to 3.10$)$ & $0.76(0.53$ to 1.09$)$ \\
\hline$\geq 4$ years & $0.73(0.46$ to 1.17$)$ & $0.97(0.75$ to 1.25$)$ & $1.48(0.82$ to 2.68$)$ & 0.99 (0.71 to 1.37$)$ \\
\hline \multicolumn{5}{|l|}{ Standardised daily dose $\ddagger$} \\
\hline Non-users & 1.00 & 1.00 & 1.00 & 1.00 \\
\hline Low & $0.77(0.47$ to 1.25$)$ & $0.98(0.75$ to 1.29$)$ & 1.25 (0.69 to 2.28$)$ & $0.92(0.66$ to 1.29$)$ \\
\hline Medium & $1.01(0.61$ to 1.66$)$ & $0.97(0.71$ to 1.33$)$ & 2.00 (1.02 to 3.93$)$ & 0.95 (0.65 to 1.40$)$ \\
\hline High & $1.00(0.55$ to 1.84$)$ & $0.73(0.48$ to 1.12$)$ & 2.45 (1.12 to 5.33$)$ & 0.65 (0.40 to 1.07$)$ \\
\hline \multicolumn{5}{|c|}{$\begin{array}{l}\text { *Adjusted for age, education, marital status, alcohol use, smoking, body mass index, self-reported comorbidities, self-reported cardiovascular } \\
\text { diseases, impaired vision, depression, cognitive impairment, functional status, self-rated health, polypharmacy and for total cholesterol, } \\
\text { high-density lipoprotein cholesterol and triglyceride concentrations. } \\
\text { †Non-users, the reference group. } \\
\text { fStandardised daily dose was defined as follows: one unit of equivalent dose was based on a lipid-lowering effect of } 10 \mathrm{mg} \text { of atorvastatin } \\
\text { (fluvastatin } 80 \mathrm{mg} \text {, lovastatin } 40 \mathrm{mg} \text {, pravastatin } 40 \mathrm{mg} \text {, simvastatin } 2 \mathrm{mg} \text {, rosuvastatin } 5 \mathrm{mg}){ }^{18}{ }^{19} \text { Low dose was defined as }<2 \text { standardised } \\
\text { unit, medium dose as } 2-4 \text { standardised unit, and high dose as } \geq 4 \text { standardised unit. }\end{array}$} \\
\hline
\end{tabular}


Table 3 Association between reported statin use at baseline and institutionalisation and death, adjusted for continuous and quintiles of propensity scores $(n=1497)$

\begin{tabular}{|c|c|c|c|c|}
\hline \multirow[b]{2}{*}{ Categorisation of statin use } & \multicolumn{2}{|c|}{ Adjusted HR (95\% Cl)* } & \multicolumn{2}{|c|}{ Adjusted HR (95\% Cl)† } \\
\hline & Institutionalisation & Death & Institutionalisation & Death \\
\hline \multicolumn{5}{|l|}{ Statin exposure } \\
\hline Non-users $\ddagger$ & 1.00 & 1.00 & 1.00 & 1.00 \\
\hline Users & $1.43(0.87$ to 2.34$)$ & $0.82(0.61$ to 1.10$)$ & $1.32(0.81$ to 2.15$)$ & 0.81 (0.61 to 1.08$)$ \\
\hline \multicolumn{5}{|l|}{ Duration of statin use } \\
\hline Non-users & 1.00 & 1.00 & 1.00 & 1.00 \\
\hline $0-<4$ & $1.77(1.01$ to 3.11$)$ & $0.74(0.52$ to 1.06$)$ & 1.65 (0.95 to 2.86$)$ & 0.73 (0.51 to 1.04$)$ \\
\hline$\geq 4$ & $1.15(0.64$ to 2.08$)$ & $0.88(0.64$ to 1.22$)$ & 1.07 (0.59 to 1.91$)$ & 0.87 (0.64 to 1.20$)$ \\
\hline \multicolumn{5}{|l|}{ Standardised daily dose§ } \\
\hline Non-users & 1.00 & 1.00 & 1.00 & 1.00 \\
\hline Low & 1.17 (0.65 to 2.13$)$ & $0.85(0.61$ to 1.19$)$ & $1.10(0.60$ to 1.99$)$ & $0.84(0.60$ to 1.17$)$ \\
\hline Medium & 1.73 (0.92 to 3.27$)$ & $0.87(0.60$ to 1.28$)$ & 1.57 (0.85 to 2.93$)$ & 0.87 (0.60 to 1.27$)$ \\
\hline High & 1.71 (0.82 to 3.57$)$ & $0.66(0.41$ to 1.07$)$ & $1.56(0.75$ to 3.24$)$ & 0.65 (0.41 to 1.05$)$ \\
\hline
\end{tabular}

more severe CVDs would be even more likely to be prescribed statins. In addition, participants adherent to treatment are likely to do better, which is hard to capture. These characteristics may have overestimated or underestimated the HRs. The implications of healthy user bias (eg, unhealthy individuals will be less likely to use statins, which may indicate the benefits of statins in observational studies) and healthy tolerator bias (eg, adherence to preventative drugs including statins is associated with better outcomes in general) should also be considered. $^{39}$ In relation to statin exposure, the non-user group may include former users of statins. Moreover, it is unknown whether statins were stopped, started or the dose was changed during the follow-up. The possibility of recall bias should be considered as the assessment of CVD comorbidities and other diseases was based on self-report alone. While some covariates adjusted for in our analysis may be potential mediators of statin use, they are also important risk factors for the clinical outcomes investigated in our analysis. The modified measurements for three components of the frailty score were used in this sample.

Participation in the CHAMP study was voluntary and the clinical characteristics of participants may have differed from those of non-participants, which may have biased the sample. The study's generalisability may be limited as this sample comprised older men living in a defined geographical location. However, the response rate in the CHAMP study is similar to other comparable cohort studies of this type. ${ }^{18}$ Moreover, the use of statins in this population $(42.9 \%)$ was very similar to a random sample of older Australians aged $\geq 75$ (43\%). Finally, the findings of this study may not be applicable to older women.

Table 4 Association between reported statin use in frail versus non-frail men and institutionalisation and death

\begin{tabular}{|c|c|c|}
\hline & Unadjusted HR $(95 \% \mathrm{Cl})(\mathrm{n}=1631)$ & Adjusted HR $(95 \% \mathrm{Cl})^{*}(\mathrm{n}=1497)$ \\
\hline \multicolumn{3}{|l|}{ Institutionalisation } \\
\hline Non-frail participants not on statins $†$ & 1.00 & 1.00 \\
\hline Non-frail participants on statins & $0.93(0.60$ to 1.44$)$ & $1.43(0.81$ to 2.51$)$ \\
\hline Frail participants not on statins & 4.58 (2.82 to 7.44$)$ & 2.07 (1.11 to 3.86$)$ \\
\hline Frail participants on statins & 5.47 (3.11 to 9.61$)$ & 4.34 (2.02 to 9.33$)$ \\
\hline \multicolumn{3}{|l|}{ Death } \\
\hline Non-frail participants not on statins & 1.00 & 1.00 \\
\hline Non-frail participants on statins & $1.05(0.83$ to 1.35$)$ & $0.90(0.66$ to 1.23$)$ \\
\hline Frail participants not on statins & 3.40 (2.49 to 4.65$)$ & 1.53 (1.03 to 2.28$)$ \\
\hline Frail participants on statins & 3.01 (1.97 to 4.61$)$ & $1.24(0.71$ to 2.17$)$ \\
\hline
\end{tabular}


In this prospective observational study, the use of statins was not associated with a significantly increased risk of institutionalisation or death. However, in this sample, frail men were more likely to be institutionalised and die than non-frail men, independent of their statin exposure. Given the wide use of statins in older adults, a regular clinical review of any observed or potential risks and benefits of statin therapy should be performed with older patients. Further longitudinal studies are warranted to confirm these associations in older women and in populations of older people across different settings. Finally, these findings call for pragmatic real-world trials specifically tailored for older frail people to examine the impact of statins on institutionalisation and other important clinical endpoints.

\section{Author affiliations}

${ }^{1}$ Faculty of Pharmacy, University of Sydney, Sydney, Australia

${ }^{2}$ Centre for Education and Research on Ageing, University of Sydney and Concord RG Hospital, Sydney, New South Wales, Australia

${ }^{3}$ Departments of Clinical Pharmacology and Aged Care, Royal North Shore Hospital, Sydney, New South Wales, Australia.

${ }^{4}$ Sydney Medical School, University of Sydney, Sydney, New South Wales, Australia

${ }^{5}$ ANZAC Institute, Concord Hospital, Sydney, New South Wales, Australia

${ }^{6}$ Departments of Medicine and Biostatistics, Boston University, Boston,

Massachusetts, USA

${ }^{7}$ Department of Intramural Research, The Sax Institute, Sydney, New South Wales, Australia

${ }^{8}$ Sydney School of Public Health, University of Sydney, Sydney, New South Wales, Australia

Contributors DG, DGLC and SNH conceived the study and were responsible for the design of the study. DG was responsible for undertaking the data analysis and drafting the manuscript. FMB, TT and KR provided input into the data analysis. All authors critically revised the drafted manuscript and have approved the final manuscript.

Funding The CHAMP study is funded by the NHMRC Project Grant (No. 301916), Sydney Medical School Foundation and Ageing and Alzheimer's Research Foundation. The sponsor had no role in the study design, data collection, data analysis, data interpretation, writing of this report or the decision to submit for publication. DG was supported by the Australian National Health and Medical Research Council (NHMRC) Early Career Fellowship.

Competing interests None.

Ethical approval The study was approved by the Sydney South West Area Health Service Human Research Ethics Committee Concord Repatriation General Hospital, Sydney, Australia.

Data sharing statement No additional data are available.

\section{REFERENCES}

1. Morgan TK, Williamson M, Pirotta $M$, et al. A national census of medicines use: a 24-hour snapshot of Australians aged 50 years and older. Med J Aust 2012;196:50-3.

2. Taylor F, Ward $\mathrm{K}$, Moore $\mathrm{TH}$, et al. Statins for the primary prevention of cardiovascular disease. Cochrane Database Syst Rev 2011; CD004816.

3. Brugts JJ, Yetgin T, Hoeks SE, et al. The benefits of statins in people without established cardiovascular disease but with cardiovascular risk factors: meta-analysis of randomised controlled trials. BMJ 2009;338:b2376.

4. Konrat C, Boutron I, Trinquart L, et al. Underrepresentation of elderly people in randomised controlled trials. The example of trials of 4 widely prescribed drugs. PLOS ONE 2012;7:e33559.
5. Vandenbroucke JP. Observational research, randomised trials, and two views of medical science. PLoS Med 2008;5:e67.

6. McLean AJ, Le Couteur DG. Aging biology and geriatric clinical pharmacology. Pharmacol Rev 2004;56:163-84.

7. Gnjidic D, Hilmer SN. Use of potentially inappropriate medications in the care of frail older people. Aging Health 2010;6:705-16.

8. Fried LP, Tangen CM, Walston J, et al. Frailty in older adults: evidence for a phenotype. J Gerontol A Biol Sci Med Sci 2001;56:M146-56.

9. Gnjidic D, Hilmer SN, Blyth F, et al. High risk prescribing and incidence of frailty among older community-dwelling men. Clin Pharmacol Ther 2012;91:521-8.

10. Gnjidic D, Hilmer S, Le Couteur D. High risk prescribing in older adults: more harm than good? Aging Health 2012;8:325-7.

11. Mondillo S, Ballo P, Barbati R, et al. Effects of simvastatin on walking performance and symptoms of intermittent claudication in hypercholesterolemic patients with peripheral vascular disease. Am J Med 2003;114:359-64.

12. Mohler ER 3rd, Hiatt WR, Creager MA. Cholesterol reduction with atorvastatin improves walking distance in patients with peripheral arterial disease. Circulation 2003;108:1481-6.

13. Gray SL, Boudreau RM, Newman AB, et al. Angiotensin-converting enzyme inhibitor and statin use and incident mobility limitation in community-dwelling older adults: the health, aging and body composition study. J Am Geriatr Soc 2011;59:2226-32.

14. LaCroix AZ, Gray SL, Aragaki A, et al. Statin use and incident frailty in women aged 65 years or older: prospective findings from the Women's Health Initiative Observational Study. J Gerontol A Biol Sci Med Sci 2008:63:369-75.

15. Hilmer SN, Gnjidic D. Statins in older adults. Aust Prescr 2013; In press.

16. Golomb BA, Evans MA, Dimsdale JE, et al. Effects of statins on energy and fatigue with exertion: results from a randomized controlled trial. Arch Intern Med 2012.

17. Grundy SM. Statin therapy in older persons: pertinent issues. Arch Intern Med 2002;162:1329-31.

18. Cumming RG, Handelsman D, Seibel MJ, et al. Cohort profile: the Concord Health and Ageing in Men Project (CHAMP). Int $J$ Epidemiol 2009;38:374-8.

19. Jones PH, Davidson $\mathrm{MH}$, Stein EA, et al. Comparison of the efficacy and safety of rosuvastatin versus atorvastatin, simvastatin, and pravastatin across doses (STELLAR ${ }^{\star}$ Trial). Am J Cardiol 2003;92:152-60.

20. Jones P, Kafonek S, Laurora I, et al. Comparative dose efficacy study of atorvastatin versus simvastatin, pravastatin, lovastatin, and fluvastatin in patients with hypercholesterolemia (the CURVES study). Am J Cardiol 1998;81:582-7.

21. Gnjidic D, Hilmer SN, Blyth F, et al. Polypharmacy cutoff and outcomes: five or more medicines were used to identify community-dwelling older men at risk of different adverse outcomes. J Clin Epidemiology 2012;65:989-95.

22. Bailey IL, Lovie JE. New design principles for visual acuity letter charts. Am J Optom Physiol Opt 1976;53:740-5.

23. Yesavage JA, Brink TL, Rose TL, et al. Development and validation of a geriatric depression screening scale: a preliminary report. J Psychiatr Res 1982;17:37-49.

24. Gnjidic D, Le Couteur DG, Naganathan V, et al. Effects of Drug Burden Index on cognitive function in older men. J Clin Psychopharmacol 2012;32:218-24.

25. Katz S, Downs TD, Cash HR, et al. Progress in development of the index of ADL. Gerontologist 1970;10:20-30.

26. Fillenbaum GG, Smyer MA. The development, validity, and reliability of the OARS multidimensional functional assessment questionnaire. J Gerontol 1981;36:428-34.

27. Rochat S, Cumming RG, Blyth F, et al. Frailty and use of health and community services by community-dwelling older men: the Concord Health and Ageing in Men Project. Age Ageing 2010;39:228-33.

28. Blyth FM, Rochat S, Cumming RG, et al. Pain, frailty and comorbidity on older men: the CHAMP study. Pain 2008;140:224-30.

29. Rosenbaum PR, Rubin DB. The central role of the propensity score in observational studies for causal effects. Biometrika 1983;70:41-55.

30. Hackam DG, Wu F, Li P, et al. Statins and renovascular disease in the elderly: a population-based cohort study. Eur Heart $J$ 2011;32:598-610.

31. Studenski S, Perera S, Patel K, et al. Gait speed and survival in older adults. JAMA 2011;305:50-8.

32. Cooper R, Kuh D, Hardy R. Objectively measured physical capability levels and mortality: systematic review and meta-analysis. $B M J$ 2010;341:c4467.

33. Olafsdottir E, Aspelund T, Sigurdsson G, et al. Effects of statin medication on mortality risk associated with type 2 diabetes in older 
persons: the population-based AGES-Reykjavik Study. BMJ Open 2011;1:e000132.

34. Shepherd J, Blauw GJ, Murphy MB, et al. Pravastatin in elderly individuals at risk of vascular disease (PROSPER): a randomised controlled trial. Lancet 2002;360:1623-30.

35. Rockwood K, Song X, MacKnight C, et al. A global clinical measure of fitness and frailty in elderly people. CMAJ 2005;173:489-95.

36. Kane RL, Shamliyan T, Talley K, et al. The association between geriatric syndromes and survival. J Am Geriatr Soc 2012;60:896-904.
37. Travison TG, Nguyen $\mathrm{AH}$, Naganathan $\mathrm{V}$, et al. Changes in reproductive hormone concentrations predict the prevalence and progression of the frailty syndrome in older men: the Concord Health and Ageing in Men Project. J Clin Endocrinol Metab 2011;96:2464-74.

38. Austin PC. The performance of different propensity-score methods for estimating relative risks. J Clin Epidemiol 2008; 61:537-45.

39. Golomb BA. Do statins reduce the risk of infection? BMJ 2011;343: d7134. 